\title{
A Study of Some Integral Problems Using Maple
}

\author{
Chii-Huei Yu \\ Department of Management and Information, Nan Jeon University of Science and Technology, Tainan City, 73746, Taiwan \\ *Corresponding Author: chiihuei@mail.njtc.edu.tw
}

Copyright (c) 2014 Horizon Research Publishing All rights reserved.

\begin{abstract}
This paper takes the mathematical software Maple as the auxiliary tool to study four types of integrals. We can obtain the Fourier series expansions of these four types of integrals by using integration term by term theorem. On the other hand, we provide two examples to do calculation practically. The research methods adopted in this study involved finding solutions through manual calculations and verifying these solutions by using Maple.
\end{abstract}

Keywords Integrals, Fourier Series Expansions, Integration Term By Term Theorem, Maple

\section{Introduction}

In calculus and engineering mathematics courses, we learnt many methods to solve the integral problems including change of variables method, integration by parts method, partial fractions method, trigonometric substitution method, and so on. In this paper, we mainly study the following four types of integrals which are not easy to obtain their answers using the methods mentioned above.

$$
\begin{aligned}
& \int \sin [r \cos (a t+b)] \cosh [r \sin (a t+b)] d t \\
& \int \cos [r \cos (a t+b)] \sinh [r \sin (a t+b)] d t \\
& \int \cos [r \cos (a t+b) \cosh [r \sin (a t+b)] d t \\
& \int \sin [r \cos (a t+b) \sinh [r \sin (a t+b)] d t
\end{aligned}
$$

Where $r, a, b$ are real numbers, $a \neq 0$. We can obtain the Fourier series expansions of these four types of integrals by using integration term by term theorem; these are the major results of this paper (i.e., Theorems 1,2 ). As for the study of related integral problems can refer to [1-15]. On the other hand, we propose some integrals to do calculation practically. The research methods adopted in this study involved finding solutions through manual calculations and verifying these solutions by using Maple. This type of research method not only allows the discovery of calculation errors, but also helps modify the original directions of thinking from manual and Maple calculations.
For this reason, Maple provides insights and guidance regarding problem-solving methods.

\section{Main Results}

Firstly, we introduce a notation and some formulas used in this study.

\subsection{Notation}

Let $z=a+i b$ be a complex number, where $i=\sqrt{-1}$, $a, b$ are real numbers. We denote $a$ the real part of $z$ by $\operatorname{Re}(z)$, and $b$ the imaginary part of $z$ by $\operatorname{Im}(z)$.

\subsection{Formulas}

\subsubsection{Euler's formula}

$$
e^{i \theta}=\cos \theta+i \sin \theta \text {, where } \theta \text { is any real number. }
$$

\subsubsection{DeMoivre's formula}

$(\cos \theta+i \sin \theta)^{n}=\cos n \theta+i \sin n \theta$, where $n$ is any integer, $\theta$ is any real number.

$$
\begin{aligned}
& \text { 2.2.3. }([16, \mathrm{p} 25]) \\
& \quad \sin (u+i v)=\sin u \cosh v+i \cos u \sinh v, \quad \text { where }
\end{aligned}
$$
$u, v$ are real numbers.

$$
\text { 2.2.4. }([16, \mathrm{p} 25])
$$

$\cos (u+i v)=\cos u \cosh v-i \sin u \sinh v$, where $u, v$ are real numbers.

2.2.5. ([16, p63])

$\sin z=\sum_{k=0}^{\infty}(-1)^{k} \frac{1}{(2 k+1) !} z^{2 k+1}$, where $z \quad$ is any complex number.

2.2.6. ([16, p63])

$\cos z=\sum_{k=0}^{\infty}(-1)^{k} \frac{1}{(2 k) !} z^{2 k}$, where $z$ is any complex number. 
Next, we introduce an important theorem used in this paper.

\subsection{Integration term by term theorem ([17, p269])}

Suppose $\left\{g_{k}(t)\right\}_{k=0}^{\infty}$ is a sequence of Lebesgue integrable functions defined on an inteval $[c, d]$. If $\sum_{k=0}^{\infty} \int_{c}^{d} \mid g_{k}(t) d t$

is convergent, then $\int_{c}^{d} \sum_{k=0}^{\infty} g_{k}(t) d t=\sum_{k=0}^{\infty} \int_{c}^{d} g_{k}(t) d t$. The following is the first major result of this study, we obtain the Fourier series expansions of the integrals (1) and (2).

\subsection{Theorem 1}

Assume $r, a, b, c$ are real numbers, $a \neq 0$. Then there exists a constant $C_{1}$ such that for all $x \in R$, the integral

$$
\begin{array}{r}
\int_{c}^{x} \sin [r \cos (a t+b)] \cosh [r \sin (a t+b)] d t \\
=\frac{1}{a} \cdot \sum_{k=0}^{\infty}(-1)^{k} \frac{r^{2 k+1}}{(2 k+1) !(2 k+1)} \sin [(2 k+1)(a x+b)]+C_{1}
\end{array}
$$

And there exists a constant $C_{2}$ such that for all $x \in R$, the integral

$$
\begin{array}{r}
\int_{c}^{x} \cos [r \cos (a t+b)] \sinh [r \sin (a t+b)] d t \\
=\frac{-1}{a} \cdot \sum_{k=0}^{\infty}(-1)^{k} \frac{r^{2 k+1}}{(2 k+1) !(2 k+1)} \cos [(2 k+1)(a x+b)]+C_{2}
\end{array}
$$

\subsubsection{Proof}

Because

$$
\begin{array}{r}
\sin [r \cos (a x+b)] \cosh [r \sin (a x+b)] \\
=\operatorname{Re}\{\sin [r \exp i(a x+b)]\}
\end{array}
$$

(By Formula 2.2.3)

$$
=\operatorname{Re}\left[\sum_{k=0}^{\infty}(-1)^{k} \frac{1}{(2 k+1) !}[r \exp i(a x+b)]^{2 k+1}\right]
$$

(Using Formula 2.2.5)

$$
=\operatorname{Re}\left[\sum_{k=0}^{\infty}(-1)^{k} \frac{r^{2 k+1}}{(2 k+1) !} \exp i[(2 k+1)(a x+b)]\right]
$$

(By DeMoivre's formula)

$$
=\sum_{k=0}^{\infty}(-1)^{k} \frac{r^{2 k+1}}{(2 k+1) !} \cos [(2 k+1)(a x+b)]
$$

(By Euler's formula)
Thus, for all $x \in R$, the integral

$$
\begin{aligned}
& \int_{c}^{x} \sin [r \cos (a t+b)] \cosh [r \sin (a t+b)] d t \\
= & \int_{c}^{x} \sum_{k=0}^{\infty}(-1)^{k} \frac{r^{2 k+1}}{(2 k+1) !} \cos [(2 k+1)(a t+b)] d t \\
= & \sum_{k=0}^{\infty}(-1)^{k} \frac{r^{2 k+1}}{(2 k+1) !} \int_{c}^{x} \cos [(2 k+1)(a t+b)] d t
\end{aligned}
$$

(By integration term by term theorem)

$$
=\frac{1}{a} \cdot \sum_{k=0}^{\infty}(-1)^{k} \frac{r^{2 k+1}}{(2 k+1) !(2 k+1)} \sin [(2 k+1)(a x+b)]+C_{1}
$$

Where $C_{1}$ is some constant.

On the other hand, using Euler's formula, DeMoivre's formula and Formula 2.2.3, 2.2.5, we have

$\cos [r \cos (a x+b)] \sinh [r \sin (a x+b)]$

$$
\begin{aligned}
& =\operatorname{Im}\{\sin [r \exp i(a x+b)]\} \\
& =\sum_{k=0}^{\infty}(-1)^{k} \frac{r^{2 k+1}}{(2 k+1) !} \sin [(2 k+1)(a x+b)]
\end{aligned}
$$

Therefore, by integration term by term theorem, we can show that there exists a constant $C_{2}$ such that for all $x \in R$, the integral

$$
\begin{array}{r}
\int_{c}^{x} \cos [r \cos (a t+b)] \sinh [r \sin (a t+b)] d t \\
=\frac{-1}{a} \cdot \sum_{k=0}^{\infty}(-1)^{k} \frac{r^{2 k+1}}{(2 k+1) !(2 k+1)} \cos [(2 k+1)(a x+b)]+C_{2} \\
\text { q.e.d. }
\end{array}
$$

\subsection{Remark 1}

In Theorem 1, because for each $x \in R$,

$$
\begin{array}{r}
\sum_{k=0}^{\infty} \int_{c}^{x}\left|(-1)^{k} \frac{r^{2 k+1}}{(2 k+1) !} \cos [(2 k+1)(a t+b)]\right| d t \\
\leq \frac{1}{|a|} \cdot \sum_{k=0}^{\infty} \frac{|r|^{2 k+1}}{(2 k+1) !(2 k+1)} \int_{c}^{x}|\cos [(2 k+1)(a t+b)]| d t \\
\leq \frac{1}{|a|} \cdot \sum_{k=0}^{\infty} \frac{|r|^{2 k+1}|x-c|}{(2 k+1) !(2 k+1)}<\infty
\end{array}
$$

It follows that we can use integration term by term theorem to show that (5) holds. The same reason that we can prove (6) by using integration term by term theorem.

Next, we determine the Fourier series expansions of the integrals (3) and (4).

\subsection{Theorem 2}

If the assumptions are the same as Theorem 1. Then there 
exists a constant $C_{3}$ such that for all $x \in R$, the integral

$$
\begin{array}{r}
\int_{c}^{x} \cos [r \cos (a t+b)] \cosh [r \sin (a t+b)] d t \\
=x+\frac{1}{a} \cdot \sum_{k=1}^{\infty}(-1)^{k} \frac{r^{2 k}}{(2 k) ! 2 k} \sin [(2 k)(a x+b)]+C_{3}
\end{array}
$$

And there exists a constant $C_{4}$ such that for all $x \in R$, the integral

$$
\begin{array}{r}
\int_{c}^{x} \sin [r \cos (a t+b)] \sinh [r \sin (a t+b)] d t \\
=\frac{1}{a} \cdot \sum_{k=1}^{\infty}(-1)^{k} \frac{r^{2 k}}{(2 k) ! 2 k} \cos [(2 k)(a x+b)]+C_{4} \quad(10)
\end{array}
$$

\subsubsection{Proof}

By Euler's formula, DeMoivre's formula and Formula 2.2.4, 2.2.6, we have

$$
\begin{array}{r}
\cos [r \cos (a x+b)] \cosh [r \sin (a x+b)] \\
=\operatorname{Re}\{\cos [r \exp i(a x+b)]\} \\
=\sum_{k=0}^{\infty}(-1)^{k} \frac{r^{2 k}}{(2 k) !} \cos [(2 k)(a x+b)] \\
=1+\sum_{k=1}^{\infty}(-1)^{k} \frac{r^{2 k}}{(2 k) !} \cos [(2 k)(a x+b)]
\end{array}
$$

Thus, for all $x \in R$, the integral

$$
\begin{array}{r}
\int_{c}^{x} \cos [r \cos (a t+b)] \cosh [r \sin (a t+b)] d t \\
=x-c+\sum_{k=1}^{\infty}(-1)^{k} \frac{r^{2 k}}{(2 k) !} \int_{c}^{x} \cos [(2 k)(a t+b)] d t
\end{array}
$$

(By integration term by term theorem)

$$
=x+\frac{1}{a} \cdot \sum_{k=1}^{\infty}(-1)^{k} \frac{r^{2 k}}{(2 k) ! 2 k} \sin [(2 k)(a x+b)]+C_{3}
$$

Where $C_{3}$ is some constant.

Similarly, by Euler's formula, DeMoivre's formula and Formula 2.2.4, 2.2.6, we obtain

$$
\begin{array}{r}
\sin [r \cos (a x+b)] \sinh [r \sin (a x+b)] \\
=-\operatorname{Im}\{\cos [r \exp i(a x+b)]\} \\
=-\sum_{k=1}^{\infty}(-1)^{k} \frac{r^{2 k}}{(2 k) !} \sin [(2 k)(a x+b)]
\end{array}
$$

By integration term by term theorem, it follows that for all $x \in R$, the integral

$$
\int_{c}^{x} \sin [r \cos (a t+b)] \sinh [r \sin (a t+b)] d t
$$

$$
=\frac{1}{a} \cdot \sum_{k=1}^{\infty}(-1)^{k} \frac{r^{2 k}}{(2 k) ! 2 k} \cos [(2 k)(a x+b)]+C_{4}
$$

Where $C_{4}$ is some constant.

q.e.d.

\subsection{Remark 2}

In Theorem 2, the reason that we can use integration term by term theorem to prove (9) and (10) is the same as Remark 1.

\section{Examples}

In the following, for the four types of integrals in this study, we propose some integrals and use Theorems 1, 2 to determine their Fourier series expansions. In addition, we evaluate some definite integrals and employ Maple to calculate the approximations of these definite integrals and their solutions for verifying our answers.

\subsection{Example 1}

In Theorem 1, taking $r=5, a=2, b=\pi / 3$ into (5), we obtain the following integral

$$
\begin{aligned}
& \int_{c}^{x} \sin \left[5 \cos \left(2 t+\frac{\pi}{3}\right)\right] \cosh \left[5 \sin \left(2 t+\frac{\pi}{3}\right)\right] d t \\
= & \frac{1}{2} \cdot \sum_{k=0}^{\infty}(-1)^{k} \frac{5^{2 k+1}}{(2 k+1) !(2 k+1)} \sin \left[(2 k+1)\left(2 x+\frac{\pi}{3}\right)\right]+C_{1}
\end{aligned}
$$

Thus, we can determine the definite integral from $t=\pi / 12$ to $t=\pi / 6$,

$$
\begin{array}{r}
\int_{\pi / 12}^{\pi / 6} \sin \left[5 \cos \left(2 t+\frac{\pi}{3}\right)\right] \cosh \left[5 \sin \left(2 t+\frac{\pi}{3}\right)\right] d t \\
=\frac{1}{2} \cdot \sum_{k=0}^{\infty}(-1)^{k} \frac{5^{2 k+1}}{(2 k+1) !(2 k+1)} \sin \frac{(4 k+2) \pi}{3} \\
-\frac{1}{2} \cdot \sum_{k=0}^{\infty}(-1)^{k} \frac{5^{2 k+1}}{(2 k+1) !(2 k+1)} \sin \frac{(2 k+1) \pi}{2}
\end{array}
$$

We use Maple to verify the correctness of (14).

$>\operatorname{evalf}(\operatorname{int}(\sin (5 * \cos (2 * \mathrm{t}+\mathrm{Pi} / 3)) * \cosh (5 * \sin (2 * \mathrm{t}+\mathrm{Pi} / 3)), \mathrm{t}=$ $\mathrm{Pi} / 12 . . \mathrm{Pi} / 6), 14)$;

$$
-11.041067811822+0 . \mathrm{I}
$$

$>\operatorname{evalf}\left(1 / 2 * \operatorname{sum}\left((-1)^{\wedge} \mathrm{k}^{*} 5^{\wedge}(2 * \mathrm{k}+1) /((2 * \mathrm{k}+1) ! *(2 * \mathrm{k}+1)) * \mathrm{~s}\right.\right.$ in $((4 * \mathrm{k}+2) * \mathrm{Pi} / 3), \mathrm{k}=0$.. infinity $)-$

$1 / 2^{*} \operatorname{sum}\left((-1)^{\wedge} \mathrm{k}^{*} 5^{\wedge}(2 * \mathrm{k}+1) /((\right.$

$2 * \mathrm{k}+1) ! *(2 * \mathrm{k}+1)) * \sin ((2 * \mathrm{k}+1) * \mathrm{Pi} / 2), \mathrm{k}=0$..infinity $), 14)$;

$$
-11.041067811821+0 . \mathrm{I}
$$

The above answers obtained by Maple appears I 
$(=\sqrt{-1})$, it is because Maple calculates by using special functions built in. Both the imaginary parts of the above answers are zero, so can be ignored.

On the other hand, in Theorem 1, taking $r=\sqrt{2}, a=5, b=-3 \pi / 4$ into (6), we have the following integral

$$
\begin{aligned}
& \int_{c}^{x} \cos \left[\sqrt{2} \cos \left(5 t-\frac{3 \pi}{4}\right)\right] \sinh \left[\sqrt{2} \sin \left(5 t-\frac{3 \pi}{4}\right)\right] d t \\
= & \frac{-1}{5} \cdot \sum_{k=0}^{\infty}(-1)^{k} \frac{(\sqrt{2})^{2 k+1}}{(2 k+1) !(2 k+1)} \cos \left[(2 k+1)\left(5 x-\frac{3 \pi}{4}\right)\right]+C_{2}
\end{aligned}
$$

Hence, we can determine the definite integral from

$$
\begin{aligned}
& t=\pi / 10 \text { to } t=2 \pi / 5, \\
& \int_{\pi / 10}^{2 \pi / 5} \cos \left[\sqrt{2} \cos \left(5 t-\frac{3 \pi}{4}\right)\right] \sinh \left[\sqrt{2} \sin \left(5 t-\frac{3 \pi}{4}\right)\right] d t \\
&=\frac{-1}{5} \cdot \sum_{k=0}^{\infty}(-1)^{k} \frac{(\sqrt{2})^{2 k+1}}{(2 k+1) !(2 k+1)} \cos \frac{(6 k+3) \pi}{4} \\
&+\frac{1}{5} \cdot \sum_{k=0}^{\infty}(-1)^{k} \frac{(\sqrt{2})^{2 k+1}}{(2 k+1) !(2 k+1)} \cos \frac{(2 k+1) \pi}{4}
\end{aligned}
$$

Next, we use Maple to verify the correctness of (16).

$>$ evalf(int $(\cos (\operatorname{sqrt}(2) * \cos (5 * t-3 * \mathrm{Pi} / 4)) * \sinh (\operatorname{sqrt}(2) * \sin ($ $5 * \mathrm{t}-3 * \mathrm{Pi} / 4)), \mathrm{t}=\mathrm{Pi} / 10 . .2 * \mathrm{Pi} / 5), 14)$;

\section{$0.44168906329422+0 . \mathrm{I}$}

$>\operatorname{evalf}\left(-1 / 5^{*} \operatorname{sum}\left((-1)^{\wedge} \mathrm{k}^{*} \operatorname{sqrt}(2)^{\wedge}(2 * \mathrm{k}+1) /((2 * \mathrm{k}+1) ! *(2 * \mathrm{k}\right.\right.$ $+1$

)$)^{*} \cos ((6 * \mathrm{k}+3) * \mathrm{Pi} / 4), \mathrm{k}=0$..infinity $)+1 / 5 * \operatorname{sum}\left((-1)^{\wedge} \mathrm{k} * \mathrm{sqrt}\right.$ $(2)^{\wedge}(2 * \mathrm{k}+1) /((2 * \mathrm{k}+1) ! *(2 * \mathrm{k}+1)) * \cos ((2 * \mathrm{k}+1) * \mathrm{Pi} / 4), \mathrm{k}=0$. infinity),14);

\section{$0.44168906329424+0 . I$}

Also, both the imaginary parts of the above answers obtained by Maple are zero, so can be ignored.

\subsection{Example 2}

In Theorem 2, taking $r=4 / 3, a=7, b=5 \pi / 6$ into (9), we can determine the following integral

$$
\begin{aligned}
& \int_{c}^{x} \cos \left[\frac{4}{3} \cos \left(7 t+\frac{5 \pi}{6}\right)\right] \cosh \left[\frac{4}{3} \sin \left(7 t+\frac{5 \pi}{6}\right)\right] d t \\
= & x+\frac{1}{7} \cdot \sum_{k=1}^{\infty}(-1)^{k} \frac{(4 / 3)^{2 k}}{(2 k) ! 2 k} \sin \left[(2 k)\left(7 x+\frac{5 \pi}{6}\right)\right]+C_{3}
\end{aligned}
$$

Thus, we obtain the definite integral from $t=\pi / 14$ to $t=2 \pi / 7$,

$$
\begin{array}{r}
\int_{\pi / 14}^{2 \pi / 7} \cos \left[\frac{4}{3} \cos \left(7 t+\frac{5 \pi}{6}\right)\right] \cosh \left[\frac{4}{3} \sin \left(7 t+\frac{5 \pi}{6}\right)\right] d t \\
=\frac{3 \pi}{14}+\frac{1}{7} \cdot \sum_{k=1}^{\infty}(-1)^{k} \frac{(4 / 3)^{2 k}}{(2 k) ! 2 k} \sin \frac{5 k \pi}{3} \\
-\frac{1}{7} \cdot \sum_{k=1}^{\infty}(-1)^{k} \frac{(4 / 3)^{2 k}}{(2 k) ! 2 k} \sin \frac{2 k \pi}{3}
\end{array}
$$

We use Maple to verify the correctness of (18) as follows:

$>$ evalf(int $(\cos (4 / 3 * \cos (7 * t+5 * \mathrm{Pi} / 6)) * \cosh (4 / 3 * \sin (7 * \mathrm{t}+5$

*Pi/6)),t=Pi/14..2*Pi/7),14);

$$
0.78316978454223+0 . \mathrm{I}
$$

$>\operatorname{evalf}\left(3 * \mathrm{Pi} / 14+1 / 7 * \operatorname{sum}\left((-1)^{\wedge} \mathrm{k}^{*}(4 / 3)^{\wedge}(2 * \mathrm{k}) /((2 * \mathrm{k}) ! *(2 *\right.\right.$ $\mathrm{k}))^{*} \sin \left(5^{*} \mathrm{k} * \mathrm{Pi} / 3\right), \mathrm{k}=1$..infinity $)-1 / 7 * \operatorname{sum}\left((-1)^{\wedge} \mathrm{k} *(4 / 3)^{\wedge}(2 * \mathrm{k}\right.$ )$/((2 * \mathrm{k}) ! *(2 * \mathrm{k})) * \sin (2 * \mathrm{k} * \mathrm{Pi} / 3), \mathrm{k}=1$..infinity $), 14)$;

\section{$0.78316978454221+0 . \mathrm{I}$}

Also, both the imaginary parts of the above answers obtained by Maple are zero, so can be ignored.

On the other hand, in Theorem 2, if taking $r=9, a=4, b=-2 \pi / 3$ into (10), we obtain the following integral

$$
\begin{aligned}
& \int_{c}^{x} \sin \left[9 \cos \left(4 t-\frac{2 \pi}{3}\right)\right] \sinh \left[9 \sin \left(4 t-\frac{2 \pi}{3}\right)\right] d t \\
= & \frac{1}{4} \cdot \sum_{k=1}^{\infty}(-1)^{k} \frac{9^{2 k}}{(2 k) ! 2 k} \cos \left[2 k\left(4 x-\frac{2 \pi}{3}\right)\right]+C_{4}
\end{aligned}
$$

Therefore, we have the definite integral from $t=\pi / 6$ to $t=\pi / 3$,

$$
\begin{array}{r}
\int_{\pi / 6}^{\pi / 3} \sin \left[9 \cos \left(4 t-\frac{2 \pi}{3}\right)\right] \sinh \left[9 \sin \left(4 t-\frac{2 \pi}{3}\right)\right] d t \\
=\frac{1}{4} \cdot \sum_{k=1}^{\infty}(-1)^{k} \frac{9^{2 k}}{(2 k) ! 2 k} \cos \frac{4 k \pi}{3} \\
-\frac{1}{4} \cdot \sum_{k=1}^{\infty}(-1)^{k} \frac{9^{2 k}}{(2 k) ! 2 k}
\end{array}
$$

Using Maple to verify the correctness of (20) as follows: $>$ evalf(int $(\sin (9 * \cos (4 * \mathrm{t}-2 * \mathrm{Pi} / 3)) * \sinh (9 * \sin (4 * \mathrm{t}-2 * \mathrm{Pi} / 3)), \mathrm{t}$ $=\mathrm{Pi} / 6 . \mathrm{Pi} / 3), 14)$;

\section{$-27.173107853571+0 . I$}

$>\operatorname{evalf}\left(1 / 4 * \operatorname{sum}\left((-1)^{\wedge} \mathrm{k}^{*} 9^{\wedge}(2 * \mathrm{k}) /((2 * \mathrm{k}) ! *(2 * \mathrm{k})) * \cos (4 * \mathrm{k} *\right.\right.$ $\mathrm{Pi} / 3), \mathrm{k}=1$..infinity $)-1 / 4 * \operatorname{sum}\left((-1)^{\wedge} \mathrm{k}^{*} 9^{\wedge}(2 * \mathrm{k}) /((2 * \mathrm{k}) ! *(2 * \mathrm{k}))\right.$, $\mathrm{k}=1$..infinity),14);

$$
-27.173107853571+3.6 \cdot 10^{-13} \mathrm{I}
$$


The imaginary parts of the above answers obtained by Maple are either zero or very small, so can be ignored.

\section{Conclusion}

From the above discussion, we know the integration term by term theorem plays a significant role in the theoretical inferences of this study. In fact, the application of this theorem is extensive, and can be used to easily solve many difficult problems; we endeavor to conduct further studies on related applications. On the other hand, Maple also plays a vital assistive role in problem-solving. In the future, we will extend the research topic to other calculus and engineering mathematics problems and solve these problems by using Maple. These results will be used as teaching materials for Maple on education and research to enhance the connotations of calculus and engineering mathematics.

\section{REFERENCES}

[1] A. A. Adams, H. Gottliebsen, S. A. Linton, and U. Martin, Automated theorem proving in support of computer algebra: symbolic definite integration as a case study, Proceedings of the 1999 International Symposium on Symbolic and Algebraic Computation, pp. 253-260, Vancouver, Canada, 1999.

[2] C. Oster, Limit of a definite integral, SIAM Review, Vol. 33, No. 1, pp. 115-116, 1991.

[3] M. A. Nyblom, On the evaluation of a definite integral involving nested square root functions, Rocky Mountain Journal of Mathematics, Vol. 37, No. 4, pp. 1301-1304, 2007.

[4] C. -H. Yu, A study on integral problems by using Maple, International Journal of Advanced Research in Computer Science and Software Engineering, Vol. 3, Issue. 7, pp. 41-46, 2013.

[5] C. -H. Yu, Evaluating some integrals with Maple,
International Journal of Computer Science and Mobile Computing, Vol. 2, Issue. 7, pp. 66-71, 2013.

[6] C.-H. Yu, Application of Maple on evaluating the closed forms of two types of integrals, Proceedings of the 17th Mobile Computing Workshop, ID16, 2012.

[7] C.-H. Yu, Application of Maple on some integral problems, Proceedings of the International Conference on Safety \& Security Management and Engineering Technology 2012, pp. 290-294, 2012.

[8] C.-H. Yu, Application of Maple on the integral problem of some type of rational functions, Proceedings of the Annual Meeting and Academic Conference for Association of IE, D357-D362, 2012.

[9] C. -H. Yu, Using Maple to study two types of integrals, International Journal of Research in Computer Applications and Robotics, Vol. 1, Issue. 4, pp. 14-22, 2013.

[10] C.-H. Yu, Application of Maple on evaluation of definite integrals, Applied Mechanics and Materials, in press.

[11] C. -H. Yu, Solving some integrals with Maple, International Journal of Research in Aeronautical and Mechanical Engineering, Vol. 1, Issue. 3, pp. 29-35, 2013.

[12] C. -H. Yu, Using Maple to study the integrals of trigonometric functions, Proceedings of the 6th IEEE/International Conference on Advanced Infocomm Technology, No. 00294, 2013.

[13] C. -H. Yu, A study of the integrals of trigonometric functions with Maple, Proceedings of the Institute of Industrial Engineers Asian Conference 2013, Springer, Vol. 1, pp. 603-, 2013.

[14] C.-H. Yu, Application of Maple on some type of integral problem, Proceedings of Ubiquitous-Home Conference 2012, pp.206-210, 2012.

[15] C.-H. Yu, Application of Maple: taking two special integral problems as examples, Proceedings of the 8th International Conference on Knowledge Community, pp.803-811, 2012.

[16] W. R. Derrick, Introductory Complex Analysis and Applications, New York: Academic Press, 1972.

[17] T. M. Apostol, Mathematical Analysis, 2nd ed., Boston: Addison-Wesley, 1975. 